\title{
Jet substructure and jet-induced medium response
}

\section{Xin-Nian Wang*}

Key Laboratory of Quark \& Lepton Physics (MOE) and Institute of Particle Physics, Central China Normal University, Wuhan 430079, China

Nuclear Science Division, Lawrence Berkeley National Laboratory, Berkeley, CA 94720, USA

E-mail: xnwang@1bl.gov

Jet structure and jet energy loss in high-energy heavy-ion collisions are investigated within the framework of the Linear Boltzmann Transport (LBT) model with special emphasis on the effect of the medium response. Inclusion of recoil medium partons is shown to reduce significantly the jet energy loss within the jet cone. They also lead to enhancement of soft hadrons in the jet fragmentation functions, enhancement of large mass groomed jets and jet transverse profile at large radius.

International Conference on Hard and Electromagnetic Probes of High-Energy Nuclear Collisions 30 September - 5 October 2018

Aix-Les-Bains, Savoie, France

${ }^{*}$ Speaker. 


\section{Introduction}

In high-energy heavy-ion collisions, jet quenching caused by parton energy loss in the quarkgluon plasma can be used as a diagnostic tool to study medium properties such as that characterized by jet transport parameters $\hat{q}$. The most studied effects of jet quenching in the first decade of high-energy heavy-ion collisions at RHIC and LHC are the suppression of single inclusive hadron spectra, dihadron correlations and $\gamma$-hadron correlations relative to the baseline spectra in $p+p$ collisions. Such suppressions are direct consequences of the suppression of leading hadrons from fragmentation of jets that have suffered from both elastic and inelastic energy loss. This suppression of leading hadrons can be described by a medium modified jet fragmentation function. The latest phenomenological study of single inclusive hadron spectra suppression at RHIC and LHC by the JET Collaboration [1] has provided the most stringent constraints on the jet transport parameters $\hat{q}$ which is used to characterize the interaction between jet and medium partons.

Medium modification of the production rate and structure of fully reconstructed jets in highenergy heavy-ion collisions can provide additional constraints on jet-medium interaction [2]. Jets are collimated clusters of hadrons within a jet cone. They are therefore determined not only by energy loss of leading partons but also influenced by how the lost energy is transported in the medium through radiated gluons and recoil medium partons. Therefore, it is necessary to include recoil partons and their further propagation in the form of jet-induced medium response in the study of jet suppression and medium modification. In this talk, I will discuss the influence of medium response in the jet suppression, medium modification of jet structures within the framework of the Linear Boltzmann Transport (LBT) model.

\section{Linear Boltzmann Transport model}

The LBT model is developed [3] to study jet transport in dense QGP medium with a special emphasis on thermal recoil partons and their further transport through the medium in the form of jet-induced medium response. The transport of both jet shower and recoil partons in the LBT model is described by the linear Boltzmann equations,

$$
\begin{gathered}
p_{a} \cdot \partial f_{a}=\int \sum_{b c d} \prod_{i=b, c, d} \frac{d^{3} p_{i}}{2 E_{i}(2 \pi)^{3}}\left(f_{c} f_{d}-f_{a} f_{b}\right)\left|\mathscr{M}_{a b \rightarrow c d}\right|^{2} \\
\times \frac{\gamma_{b}}{2} S_{2}(\hat{s}, \hat{t}, \hat{u})(2 \pi)^{4} \delta^{4}\left(p_{a}+p_{b}-p_{c}-p_{d}\right)+\text { inelastic, }
\end{gathered}
$$

where the summation is over all possible parton flavors and channels of scattering, $f_{i}=(2 \pi)^{3} \delta^{3}(\vec{p}-$ $\left.\vec{p}_{i}\right) \delta^{3}\left(\vec{x}-\vec{x}_{i}-\vec{v}_{i} t\right)(i=a, c)$ are the phase-space density for jet shower partons before and after scattering and medium recoil partons, $f_{i}=1 /\left(e^{p_{i} \cdot u / T} \pm 1\right)(i=b, d)$ are phase-space distributions for thermal partons in the QGP medium with local temperature $T$ and fluid velocity $u=(1, \vec{v}) / \sqrt{1-\vec{v}^{2}}$, and $\gamma_{b}$ is the color-spin degeneracy for parton $b$.

The leading-order (LO) elastic scattering amplitudes $\left|\mathscr{M}_{a b \rightarrow c d}\right|^{2}$ have collinear divergencies that are regularized in the LBT model by a factor $S_{2}(\hat{s}, \hat{t}, \hat{u})=\theta\left(\hat{s} \geq 2 \mu_{D}^{2}\right) \theta\left(-\hat{s}+\mu_{D}^{2} \leq \hat{t} \leq-\mu_{D}^{2}\right)$, where $\hat{s}, \hat{t}$, and $\hat{u}$ are Mandelstam variables, and $\mu_{D}^{2}=(3 / 2) g^{2} T^{2}$ is the Debye screening mass with 3 quark flavors. The corresponding elastic cross sections are $d \sigma_{a b \rightarrow c d} / d \hat{t}=\left|\mathscr{M}_{a b \rightarrow c d}\right|^{2} / 16 \pi \hat{s}^{2}$. 
The strong coupling constant $\alpha_{s}=g^{2} / 4 \pi \approx 0.15$ is fitted to experimental data. The differential inclusive rates for gluon radiation is assumed to follow that from the high-twist approach [4],

$$
\frac{d \Gamma_{a}^{\text {inel }}}{d z d k_{\perp}^{2}}=\frac{6 \alpha_{s} P_{a}(z) k_{\perp}^{4}}{\pi\left(k_{\perp}^{2}+z^{2} m^{2}\right)^{4}} \frac{p \cdot u}{p_{0}} \hat{q}_{a}(x) \sin ^{2} \frac{\tau-\tau_{i}}{2 \tau_{f}},
$$

where $P_{a}(z)$ is the splitting function for parton $a$ to emit a gluon, $a \rightarrow a+g$, with momentum fraction $z . k_{\perp}$ is the transverse momentum of the emitted gluon, $m$ is the mass of the propagating parton, $\tau_{f}=2 p_{0} z(1-z) /\left(k_{\perp}^{2}+z^{2} m^{2}\right)$ is the gluon formation time and $\tau_{i}$ is the time of the last gluon emission. The elastic scattering rate in the inelastic processes has been factorized into the jet transport coefficient,

$$
\hat{q}_{a}(x)=\sum_{b c d} \rho_{b}(x) \int d \hat{t} q_{\perp}^{2} \frac{d \sigma_{a b \rightarrow c d}}{d \hat{t}}
$$

which is defined as the transverse momentum transfer squared per mean-free-path in the local comoving frame of the QGP fluid. The parton density $\rho_{b}(x)$ includes the degeneracy factor. A Poisson distribution with the mean $\left\langle N_{g}^{a}\right\rangle=\Delta \tau \Gamma_{a}^{\text {inel }}$ is assumed to simulate multiple gluon radiations associated with each elastic scattering. Global energy and momentum conservation is ensured in each scattering with multiple radiated gluons.

During each scattering in the LBT model, the initial thermal parton $b$ is recorded as "negative" partons and they are also allowed to propagate in the medium according to the Boltzmann equation. The energy and momentum of these "negative" partons will be subtracted from all final observables to account for the back-reaction in the Boltzmann transport equations. They are part of the jetinduced medium response and manifest as the diffusion wake behind the propagating jet shower partons.

\section{Modification of single inclusive and $\gamma$ jets}

The LBT model has been used to describe both single inclusive light and heavy flavor hadron suppression [5], $\gamma$-hadron [6], $\gamma$-jet [7] and $Z^{0}$-jet correlations [8]. To study the medium modification of single inclusive and $\gamma$-jets, initial jet production in $\mathrm{p}+\mathrm{p}$ collisions is simulated with PYTHIA. The jet shower partons are transported through the QGP medium according to the LBT model with the bulk medium given by the CLVisc [9] hydrodynamic simulations.

The suppression factors for single inclusive jet spectra in $\mathrm{Pb}+\mathrm{Pb}$ collisions at $\sqrt{s}=2.76$ and $5.02 \mathrm{TeV}$ are shown in Fig. 1 (left) together with the latest ATLAS data [10]. The LBT results that are consistent with the experimental data show very weak colliding energy dependence at LHC energies despite the fact that the initial parton density at $5.02 \mathrm{TeV}$ is about $20 \%$ higher than at 2.76 $\mathrm{TeV}$. The suppression factors also show very weak transverse momentum dependence in the range of the experimental coverage. These two features are the consequences of the initial parton spectra and the transverse momentum dependence of jet energy loss.

To illustrate the colliding energy and transverse momentum dependence of the jet energy loss, we show the averaged energy loss $\left\langle\Delta p_{T}\right\rangle$ in Fig. 1(right) for leading jets in the 0-10\% most central $\mathrm{Pb}+\mathrm{Pb}$ collisions at two colliding energies, $\sqrt{s_{\mathrm{NN}}}=2.76$ and $5.02 \mathrm{TeV}$, from LBT simulations. PYTHIA simulations for initial jet production are used for jets both in $\mathrm{p}+\mathrm{p}$ collisions and initial jet shower in LBT simulations. The difference between the final transverse energies of the vacuum and 

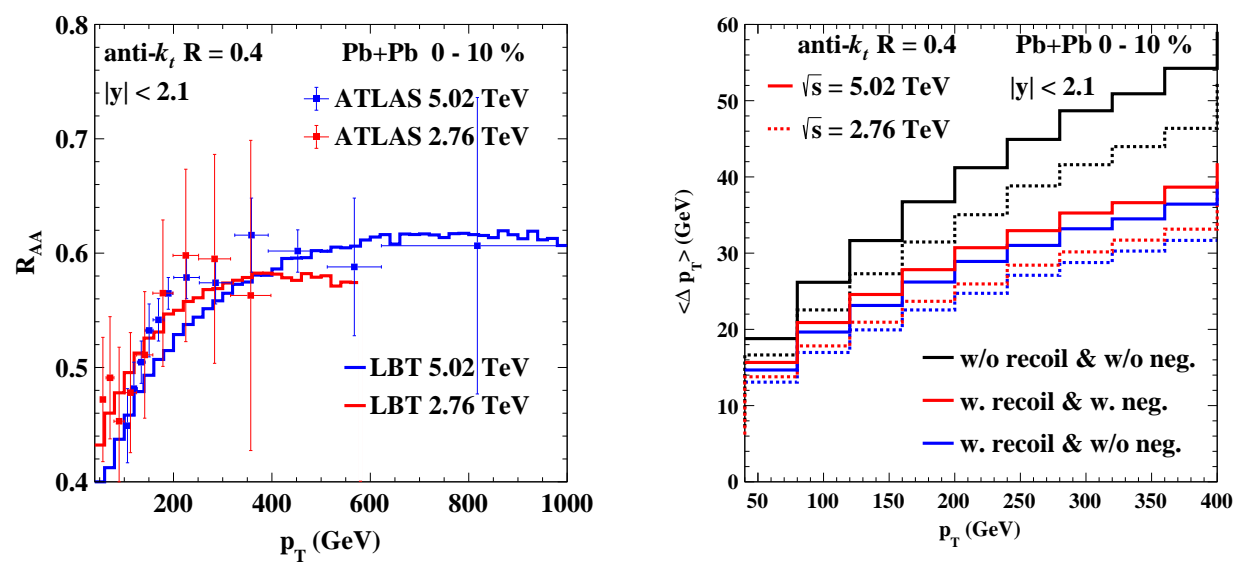

Figure 1: (Left) LBT results on $R_{\mathrm{AA}}\left(p_{T}\right)$ for single inclusive jet spectra in $0-10 \%$ central $\mathrm{Pb}+\mathrm{Pb}$ collisions at $\sqrt{s_{\mathrm{NN}}}=2.76$ and $5.02 \mathrm{TeV}$ as compared to ATLAS data [10]. (Right) Average jet transverse energy loss as a function of vacuum jet $p_{T}$ most central $10 \% \mathrm{~Pb}+\mathrm{Pb}$ collisions at (solid) $\sqrt{s_{\mathrm{NN}}}=5.02 \mathrm{GeV}$ and (dash) $2.76 \mathrm{TeV}$.

medium-modified leading jet is defined as the jet transverse energy loss as shown in Fig. 1(right) as a function of the vacuum jet transverse energy. The transverse jet energy loss at $\sqrt{s_{\mathrm{NN}}}=5.02 \mathrm{TeV}$ is about $15 \%$ larger than at $\sqrt{s_{\mathrm{NN}}}=2.76 \mathrm{TeV}$ in the $p_{T}=50-400 \mathrm{GeV} / c$ range when the medium response (recoil and "negative" partons) is taken into account. It increases with the vacuum jet transverse energy logarithmically. Such a weak $p_{T}$-dependence of the jet transverse energy loss is caused by a combination of effects due to jet-induced medium response, radial expansion and jet flavor (quarks and gluons) composition.
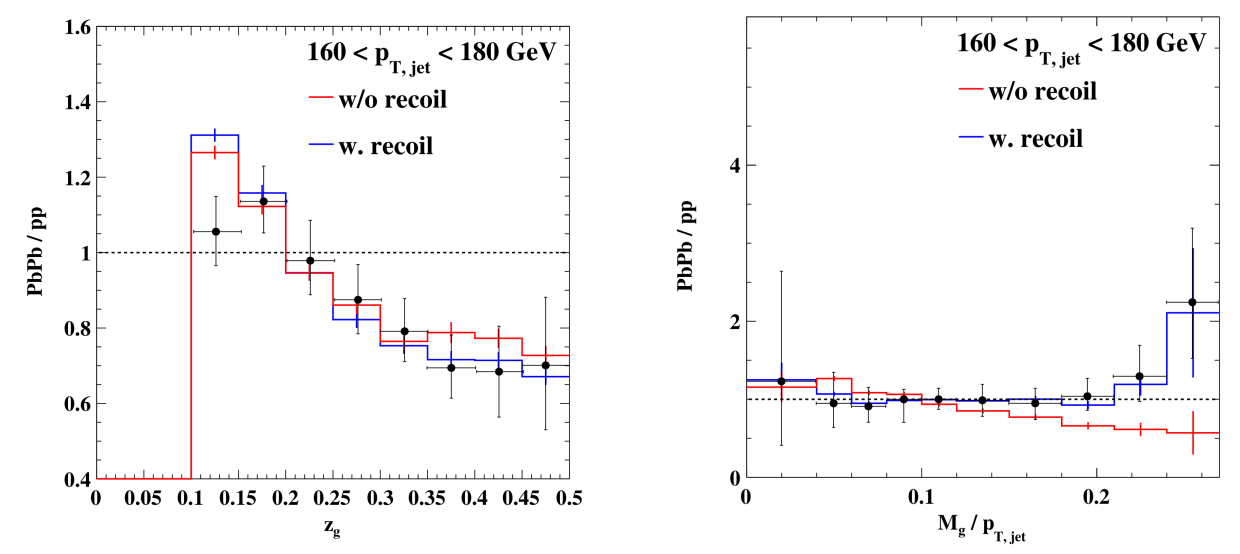

Figure 2: (Left) LBT results on medium modification of the splitting functions and (Right) mass distributions of groomed jets in central $\mathrm{Pb}+\mathrm{Pb}$ collisions at $\sqrt{s_{\mathrm{NN}}}=2.76 \mathrm{TeV}$ with and without medium recoil as compared to CMS data [12].

To further study the effect of medium response on the jet structure, we have also calculated the medium modification of the splitting functions and the mass distribution of groomed jets as shown in Fig. 2. As one can see, the effect of medium recoil which consists mostly of soft partons does not affect the hard splitting function of the groomed jets. However, the soft recoil partons do lead to the enhancement of groomed jets with large mass. These soft partons from medium recoil are also 
shown to lead to the enhancement of jet fragmentation functions at small momentum fraction $z$ [6]. The onset of the enhancement of soft hadrons is found to occur at a constant transverse momentum which reflects the thermal nature of hadrons from the medium response.
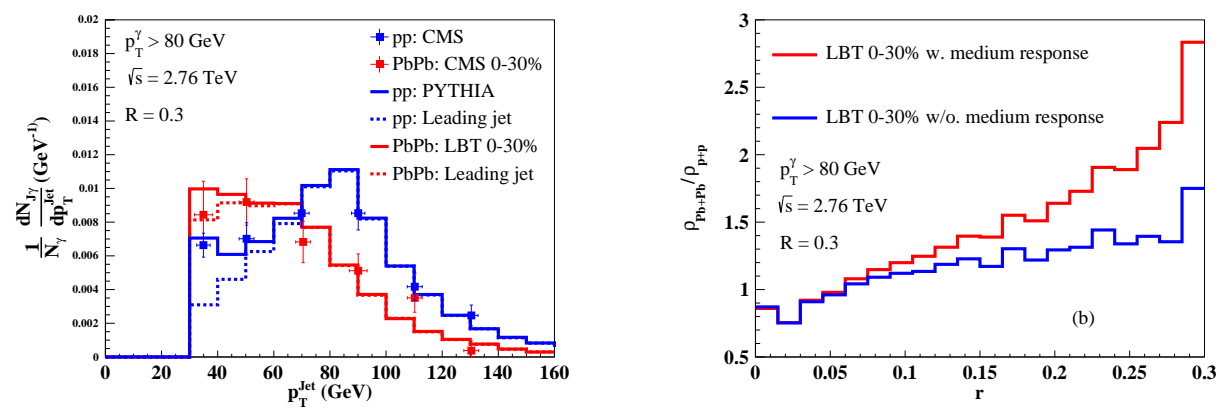

Figure 3: (Left) LBT results on $\gamma$-jet spectra in $\mathrm{p}+\mathrm{p}$ and central $\mathrm{Pb}+\mathrm{Pb}$ collisions as compared to CMS data [11] and (Right) the modification of jet transverse profile with and without medium response.

The soft hadrons from the medium response will also modify the jet transverse profile. Shown in Fig. 3(left) are the $\gamma$-triggered jet spectra in central $\mathrm{Pb}+\mathrm{Pb}$ collisions from LBT simulations as compared to CMS data [11]. One can clearly see the shift of the transverse momentum of $\gamma$-jet due to jet energy loss (including the effect of the medium recoil). These jets should also have different jet transverse profile as compared to that in $p+p$ collisions. Shown in Fig. 3(right) are the medium modification factors of the jet transverse profile with and without medium response. One can see that the jet transverse profile is significantly enhanced at large radius due to contributions from the medium response.

\section{References}

[1] K. M. Burke et al. [JET Collaboration], Phys. Rev. C 90, no. 1, 014909 (2014).

[2] I. Vitev, S. Wicks and B. W. Zhang, JHEP 0811, 093 (2008).

[3] H. Li, F. Liu, G. 1. Ma, X. N. Wang and Y. Zhu, Phys. Rev. Lett. 106, 012301 (2011); Y. He, T. Luo, X. N. Wang and Y. Zhu, Phys. Rev. C 91, 054908 (2015).

[4] X. N. Wang and X. F. Guo, Nucl. Phys. A 696, 788 (2001).

[5] S. Cao, T. Luo, G. Y. Qin and X. N. Wang, Phys. Lett. B 777, 255 (2018).

[6] W. Chen, S. Cao, T. Luo, L. G. Pang and X. N. Wang, Phys. Lett. B 777, 86 (2018).

[7] T. Luo, S. Cao, Y. He and X. N. Wang, Phys. Lett. B 782, 707 (2018).

[8] S. L. Zhang, T. Luo, X. N. Wang and B. W. Zhang, Phys. Rev. C 98, 021901 (2018).

[9] L. G. Pang, H. Petersen and X. N. Wang, Phys. Rev. C 97, no. 6, 064918 (2018).

[10] G. Aad et al. [ATLAS Collaboration], Phys. Rev. Lett. 114, no. 7, 072302 (2015); M. Aaboud et al. [ATLAS Collaboration], arXiv:1805.05635 [nucl-ex].

[11] S. Chatrchyan et al. [CMS Collaboration], Phys. Lett. B 718, 773 (2013).

[12] A. M. Sirunyan et al. [CMS Collaboration], Phys. Rev. Lett. 120, no. 14, 142302 (2018); JHEP 1810, 161 (2018). 